\title{
Dynamic model of Loan Portfolio with Lévy Asset Prices
}

\begin{abstract}
Martin Šmíd ${ }^{1}$
Abstract. We generalize the well known Merton-Vasicek (KMV) model of a loan portfolio value in two ways: we assume a Lévy process of the debtors' assets' value (instead of the Gaussian one) and we model a dynamics of the portfolio value so that the debts may last several periods (instead of a single one). Our model is computable by simulation.
\end{abstract}

Keywords: risk management, loan portfolio, dynamic model

JEL classification: G32

AMS classification: $90 \mathrm{C} 15$

\section{The Merton-Vasicek Model}

Vasicek, in his famous paper [O.A.Vasicek(2002)], assumes a large portfolio of loans. The assets $A^{i}$ of the $i$-th debtor follow the geometric Brownian motion

$$
\frac{d A_{\tau}^{i}}{A_{\tau}^{i}}=\mu d \tau+\sigma d W_{\tau}^{i}
$$

where $\mu$ and $\sigma$ are constants and $W_{t}^{i}$ is the standard Wiener process. It is further assumed that the debtor is obliged to pay a (non-random) instalment $B_{i}^{t}$ at each of the times $t \in \mathbb{N}$.

By solving (1) and subtracting the annual payment, we get

$$
a_{t}^{i}=a_{t-1}^{i}+\eta_{t}^{i}+X_{t}^{i}, \quad a_{t}^{i}=\log A_{t}^{i}, \quad \eta_{t}^{i}=\mu-\frac{1}{2} \sigma^{2}-\log B_{t}^{i}
$$

where $X_{t}^{i}=\sigma\left(W_{t}^{i}-W_{t-1}^{i}\right)$ is centred normal random variable with variance $\sigma^{2}$. Clearly, the (conditional) probability $p_{t}^{i}$ of the default of the $i$-th debtor is

$$
p_{t}^{i}=\mathbb{P}\left(a_{t}^{i}<0 \mid a_{t-1}^{i}\right)=\xi_{t}^{i}\left(-a_{t-1}^{i}-\eta_{t-1}^{i}\right)
$$

where $\xi_{t}^{i}$ is the cumulative distribution function (c.d.f.) of $X_{t}^{i}$.

Further, it is assumed by Vasicek, that

$$
\operatorname{corr}\left(X_{t}^{i}, X_{t}^{j}\right)=\rho
$$

for any $i \neq j$ and some $\rho$. It is easy to see that the latter assumption is fulfilled, for instance, if there exist mutually independent (normal) variables $Y_{t}, Z_{t}^{1}, Z_{t}^{2}, \ldots$, (independent of the evolution of the debtors' assets up to $t-1)$ such that $Z_{t}^{1}, Z_{t}^{2}, \ldots$ are equally distributed and

$$
X_{t}^{i}=Y_{t}+Z_{t}^{i}, \quad i \in \mathbb{N},
$$

such that $\operatorname{var} Y_{t}=\rho \operatorname{var} X_{t}^{1}$ and $\operatorname{var} Z_{t}^{1}=(1-\rho) \operatorname{var} X_{t}^{1}$ - to see it, note that

$$
\operatorname{corr}\left(X_{t}^{i}, X_{t}^{j}\right)=\frac{\operatorname{var} Y_{t}}{\operatorname{var} Z_{t}^{i}+\operatorname{var} Y_{t}} .
$$

Further, if the number of the debtors is very large, and if, for all $i, a_{t}^{i}=a_{t}$ and $\eta_{t}^{i}=\eta_{t}$ for some $a_{t}$ and $\eta_{t}$ (or, more generally, $p_{t}^{i}=p_{t}$ for some $p_{t}$ ) then, by the Law of Large Numbers, the percentage loss $L_{t}$ of the bank is conditionally constant given $Y_{t}, a_{t-1}$ :

$$
L_{t} \doteq \psi_{t}\left(-a_{t-1}-\eta_{t}-Y_{t}\right)
$$

\footnotetext{
${ }^{1}$ Institute of Information Theory and Automation, Academy of Sciences of the Czech Republic, e-mail: smid@utia.cas.cz
} 
where $\psi_{t}$ is the c.d.f. of $Z_{t}^{1}$ and, consequently, by the Complete Probability Theorem,

$$
\begin{gathered}
\mathbb{P}\left(L_{t}<\theta \mid p_{t}\right) \doteq 1-\phi_{t}\left(-a_{t-1}-\eta_{t}-\psi_{t}^{-1}(\theta)\right) \\
=1-\phi_{t}\left(\xi_{t}^{-1}\left(p_{t}\right)-\psi_{t}^{-1}(\theta)\right)
\end{gathered}
$$

(see [O.A.Vasicek(2002)] for details).

\section{A Generalization}

We generalize the Merton-Vasicek model two ways: we release the assumption of the normality of the factors and we take the dynamics of the system into account, making $p_{t}$ 's endogenous and no longer requiring the identical initial states of the borrowers' assets. Our goal is to describe the distribution of the losses by specifying the conditional distribution function of $L_{t}$ given

$$
\bar{L}_{t}=\left(L_{1}, L_{2}, \ldots L_{t}\right)
$$

for each $t$.

For a greater mathematical rigour, we assume the infinite number of loans in the portfolio rather than their "large amount".

To model the dynamics realistically, we assume that there is a certain amount of newly acquired deals at the beginning each period: we denote $\pi_{t}$ the $\bar{L}_{t}$-measurable variable determining the ratio of the loans new at $t$ to their overall amount (the overall amount including the new ones plus the existing ones excluding those which have defaulted at the time $t$ ). We assume the assets of a newly coming debtor to be distributed according to a common continuous and strictly increasing conditional c.d.f. $\gamma_{t}$ given $\bar{L}_{t}$ and we suppose the assets of all the newcomers be mutually conditionally independent given and $\bar{L}_{t}$.

Remark 1. We express this (more rigorously) as follows: Let $t \in \mathbb{N}$ and let, for each $i \in \mathbb{N}$, there exist a Bernoulli variable $I_{t}^{i}$ with a parameter $\pi_{t}$; if $I_{t}^{i}=1$ then a newly coming loan will be indexed by $i$ during the period from $t$ to $t+1$; if, on the other hand, $I_{t}^{i}=0$, then the first existing loan out of those which did not default at $t$ and which are still not indexed will be indexed by $i$. Naturally, $I_{t}^{1}, I_{2}^{1}, \ldots$ are required to be mutually conditionally independent given $\bar{L}_{t}$ and independent of all $Y_{\bullet}$ and $Z_{\bullet}$.

Let $t, i \in \mathbb{N}$. Coping with the reindexing, we reformulate (2) as

$$
\tilde{a}_{t}^{i}=a_{t-1}^{i}+\eta_{t}^{i}+X_{t}^{i},
$$

where $\eta_{t}^{i}$ is an arbitrary value (including the installment if there is any), $a_{t-1}^{i}$ and $\tilde{a}_{t}^{i}$ are the values of the $\log$ assets of the $i$-th debtor at the time $t-1, t$ respectively ${ }^{1}$ and $X_{t}^{i}$ is given by (4). The variables $Y_{t}$ and $Z_{t}^{1}$ do not have to be normal now but it is only required that their conditional distribution functions $\phi_{t}$ and $\psi_{t}$ are strictly monotonic and continuous. ${ }^{2}$ Later (Section 3 ) we show how (8) arises naturally from a generazitation of (1).

Naturally, we define the percentage loss as

$$
L_{t}=\lim _{n \rightarrow \infty} \frac{1}{n} \sum_{i=1}^{\infty} 1\left[\tilde{a}_{t}^{i}<0\right] .
$$

$t \in \mathbb{N}$, in our new infinite setting.

Getting to the distribution of the series $L_{1}, L_{2}, \ldots$, let us assume $a_{0}^{1}, a_{0}^{2}, \ldots$ are i.i.d (with a strictly increasing continous c.d.f. $\alpha_{0}$ ).

To describe the distribution, we shall proceed by induction:

Let $t \in \mathbb{N}$ and assume that

\footnotetext{
${ }^{1}$ Remember that a debtor may be indexed by different index in different periods; here, $i$ is the index valid from $t-1$ to $t$.

${ }^{2}$ Depending on the normallity no way, both $(7)$ and $(5)$ keep holding (with $=$ instead of $\doteq$ ) even under the generalized conditions.
} 
(1) we know the the (joint) distribution of $\bar{L}_{t-1}$

(2) the variables $a_{t-1}^{1}, a_{t-1}^{2}, \ldots$ are identically conditinally distributed and mutually conditionally independent given $\bar{L}_{t-1}$

(3) the conditional distribution function $\alpha_{t-1}$ of $a_{t-1}^{1} \mid \bar{L}_{t-1}$ is known to us.

Based on the assumptions, let us determine the distributions of $L_{t} \mid \bar{L}_{t-1}$ and $a_{t}^{\bullet} \mid \bar{L}_{t}$.

Note first that, since $Y_{t}, Z_{t}^{1}, Z_{t}^{2}, \ldots$ are independent of $\left(\bar{L}_{t-1}, a_{t-1}^{1}, a_{t-1}^{2}, \ldots\right)$, all the variables

$$
Z_{t}^{1}, a_{t-1}^{1}, Z_{t}^{2}, a_{t-1}^{2} \cdots
$$

are mutually conditionally independent given $\left(\bar{L}_{t-1}, Y_{t}\right)$. In particular, for any $i \in \mathbb{N}$, the conditional distribution of $\dot{Z}_{t}^{i}=a_{t-1}^{i}+Z_{t}^{i}$ given $\bar{L}_{t-1}$ is given by the convolution of the distributions of the summands, hence, for any $s \in \mathbb{R}$,

$$
\begin{gathered}
\mathbb{P}\left[\tilde{a}_{t}^{i}<s \mid \bar{L}_{t-1}, Y_{t}\right]=\mathbb{P}\left[\dot{Z}_{t}^{i}<s-\eta_{t}-Y_{t} \mid \bar{L}_{t-1}, Y_{t}\right]=\Psi_{t}(s) \\
\Psi_{t}(s)=\tilde{\psi}_{t}\left(s-\eta_{t}-Y_{t}\right), \quad \tilde{\psi}_{t}=\alpha_{t-1} \circ \psi_{t},
\end{gathered}
$$

and, similarly,

$$
\dot{p}_{t}=\mathbb{P}\left[\tilde{a}_{t}^{i}<0 \mid \bar{L}_{t-1}\right]=\Xi_{t}\left(-\eta_{t}\right)
$$

where $\Xi_{t}=\alpha_{t-1} \circ \psi_{t} \circ \phi_{t}$.

Moreover, $\dot{Z}_{t}^{1}, \dot{Z}_{t}^{2}, \ldots$ are mutually conditionally independent given $\left(\bar{L}_{t-1}, Y_{t}\right)$, which implies, by the Law of Large Numbers, applied to the conditional distributions of $\dot{Z}_{t}^{\bullet}$, that $L_{t}$ is conditionally constant given $\left(\bar{L}_{t-1}, Y_{t}\right)$ with

$$
L_{t}=\mathbb{P}\left[\tilde{a}_{t}^{i}<0 \mid \bar{L}_{t-1}, Y_{t}\right]=\Psi_{t}(0)
$$

Applying the Complete Probability Theorem and the independence of $\bar{L}_{t-1}$ and $Y_{t}$, we finally get

$$
\begin{aligned}
\mathbb{P}\left[L_{t} \quad<\quad\right. & \left.\theta \mid \bar{L}_{t-1}\right]=\int \mathbb{P}\left[L_{t}<\theta \mid \bar{L}_{t-1}, y\right] d \mathbb{P}_{Y_{t}}(y) \\
& =\int \mathbb{P}\left[\tilde{\psi}_{t}\left(-\eta_{t}-y\right)<\theta \mid \bar{L}_{t-1}, y\right] d \mathbb{P}_{Y_{t}}(y) \\
& =\int \mathbb{P}\left[-\tilde{\psi}_{t}^{-1}(\theta)-\eta_{t}<y \mid \bar{L}_{t-1}, y\right] d \mathbb{P}_{Y_{t}}(y) \\
& =\int_{-\eta_{t}-\tilde{\psi}_{t}^{-1}(\theta)} d \mathbb{P}_{Y_{t}}(y)=1-\phi_{t}\left(-\eta_{t}-\tilde{\psi}_{t}^{-1}(\theta)\right) \\
& =1-\phi_{t}\left(\Xi_{t}^{-1}\left(\dot{p}_{t}\right)-\tilde{\psi}_{t}^{-1}(\theta)\right) .
\end{aligned}
$$

To completely describe the distribution of the losses, it remains to specify $\alpha_{t}$ (i.e. the c.c.d.f of $a_{t}^{1}$ ) which we do in the following Lemma:

Lemma 1. It follows that $a_{t}^{1}, a_{t}^{2}, \ldots$ are conditionally independent given $\bar{L}_{t}$ and

$$
\alpha_{t}(s)=\pi_{t} \gamma_{t}(s)+\left(1-\pi_{t}\right) \zeta_{t}(s), \quad \zeta_{t}(s)=\frac{\Psi_{t}\left(s-\Psi_{t}^{-1}\left(L_{t}\right)\right)-L_{t}}{1-L_{t}} .
$$

Proof. Denote $\hat{a}_{t}^{i}$ the $i$-th existing loan which has not defaulted at $t$. We show that $\hat{a}_{t}^{1}, \hat{a}_{t}^{2}, \ldots$ are mutually conditionally independent given $\bar{L}_{t}$ with common c.c.d.f. $\zeta_{t}$; the Lemma will then follow by the textbook probability calculus. Before doing so, however, let us mention an easily provable fact:

Auxiliary assertion. Let $u$ and $v$ be independent unit uniform and let $c \in(0,1)$ be an independent random variable. Then the variable

$$
v_{u, v, c}= \begin{cases}u & \text { if } u<c \\ c+v(1-c) & \text { otherwise }\end{cases}
$$


is unit uniform independent of $c$.

Returning to the main proof, let $u_{1}, v_{1}, u_{2}, v_{2}, \ldots$ be mutually independent unit uniform variables, independent of $\bar{L}_{t}$ and of the assets of all newcomers. By [Pollard(2002)] p. 238, [Kallenberg(2002)] Theorem 6.10 and our Auxiliary assertion, the distribution of $\bar{L}_{t}, \tilde{a}_{t}^{1}, \tilde{a}_{t}^{2}, \ldots$ will not change if $\tilde{a}_{t}^{i}=\Psi_{t}^{-1}\left(v_{u_{i}, v_{i}, L_{t}}\right)$. Hence let us assume the last equality and note that

$$
\tilde{a}_{t}^{i}<0 \Leftrightarrow v_{u_{i}, v_{i}, L_{t}}<\Psi_{t}(0) \Leftrightarrow u_{i}<\Psi_{t}(0) \Leftrightarrow u_{i}<L_{t}
$$

If all $\bar{L}_{t}, u_{1}, u_{2}, \ldots$ were deterministic then, for any $i, \hat{a}_{t}^{i}=\Psi_{t}^{1}\left(L_{t}+v_{j}\left(1-L_{t}\right)\right)=\zeta_{t}^{-1}\left(v_{j}\right)$ for some $j$ i.e. $\zeta_{t}$ would be a c.d.f. of $\hat{a}_{t}^{i}$ and it could be easily checked that $\hat{a}_{t}^{1}, \hat{a}_{t}^{2}, \ldots$ are independent; however, this implies, by [Hoffmann-Jørgenson(1994)]4.5.2, that $\zeta_{t}$ is a conditional c.d.f. of $\hat{a}_{t}^{i}$ for any $i$ and that $\hat{a}_{t}^{1}, \hat{a}_{t}^{2}, \ldots$ are conditionally independent given $\bar{L}_{t}$.

By the initial assumptions, (9) and (10), we have completely described the distribution of $L$.

\section{The Geometric Lévy Assets}

In the present Section we show that our generalization is suitable in the case that the assets of the individuals follow a geometric Lévy, instead of a geometric Brownian, motion.

Before doing so, let us stay with the Brownian model for a while and note, even if it is not a necessary condition for the validity of the assumptions of the model, that it is quite natural to assume that $W^{i}=$ $U^{0}+U^{i}$ where $U^{0}$ and $U^{i}$ are independent Brownian motions (the first playing role of the common factor, the latter being the individual one) - clearly, $U^{1}, U^{2}, \ldots$ have to be equally distributed. Reflecting this and preparing for discontinuous paths, we may rewrite the evolution of the assets of the $i$-th borrower as

$$
\frac{d A_{t}^{i}}{A_{t-}^{i}}=\mu d t+d U_{t}^{0}+d U_{t}^{i},
$$

Getting back to our generalization, we no longer require $U^{0}, U^{1}, \ldots$ to be Brownian but we allow them to be Lévy processes with $\mathbb{E} U_{1}^{i}=0$, var $U_{1}^{i}<\infty$. Since the variances of both $U^{1}, U^{2}$ at the unit time are finite, so have to be their absolute moments at finite times implying the $i$-th process to possess a Lévy decomposition

$$
U_{t}^{i}=\varsigma_{i} W_{t}^{i}+J_{t}, \quad J_{t}=\int_{-\infty}^{\infty} z N^{i}(t . d z)
$$

where $W_{t}^{i}$ is a standard Wiener process $N^{i}$ is a compensated Poisson measure given by a Lévy measure $\nu_{i}$ (see e.g. [Oksendal and Sulem(2004)] or [Kallenberg(2002)] for the notions of Poisson and Lévy measures and the Lévy decomposition). Clearly $\nu_{1}=\nu_{2}=\ldots$.

Lemma 2. Under our assumptions, (8) holds with

$$
\begin{gathered}
\eta_{t}^{i}=\mu-\frac{1}{2}\left(\left|\nu_{0}\right|+\varsigma_{0}^{2}+\left|\nu_{1}\right|+\varsigma_{1}^{2}\right)-\log B_{t}^{i} \\
Y_{t}=\varsigma_{0} H_{t}^{0}+\int_{0}^{1} \int_{-\infty}^{\infty}[\log (1+s)-s] N_{t}^{0}(d s, d z) \\
Z_{t}^{i}=\varsigma_{0} H_{t}^{i}+\int_{0}^{1} \int_{-\infty}^{\infty}[\log (1+s)-s] N_{t}^{i}(d s, d z)
\end{gathered}
$$

where, for any $i \in \mathbb{N}, H_{t}^{i}$ is standard normal and $N_{t}^{i}$ is a compensated Poisson measure given by $\nu_{i}$ such that $H_{t}^{0}, N_{t}^{0}, H_{t}^{1}, N_{t}^{1}, \ldots$ are mutually independent. 
Proof. Fix $i \in \mathbb{N}$ and denote $V_{\tau}=\mu(\tau-t)+U_{\tau-t}^{0}+U_{\tau-t}^{i}, \tau \geq 0$. Clearly, $V$ is a Lévy process with Lévy decomposition

$$
\begin{gathered}
V_{\tau}=\mu t+\theta W+J_{t}, \quad \theta=\sqrt{\varsigma_{0}^{2}+\varsigma_{1}^{2}}, \quad W_{\tau}=\theta^{-1}\left(\varsigma_{0} W_{t-\tau}^{0}+\varsigma_{1} W_{\tau-t}^{i}\right), \\
J_{\tau}=J_{\tau-t}^{0}+J_{\tau-t}^{i} .
\end{gathered}
$$

Evidently, $W$ is a standard Wiener process and $J$ is a compensated Lévy jump process with Lévy measure $\nu=\nu_{0}+\nu_{i}$.

The evolution of the assets clearly fulfils

$$
\frac{d A_{t+\tau}^{i}}{A_{t+\tau-}^{i}}=d V_{\tau}
$$

with the solution, according to [Oksendal and Sulem(2004)] 1.15, given by

$$
\begin{aligned}
A_{t+\tau}= & A_{t} \exp \left\{\left(\mu-\frac{1}{2} \theta^{2}\right) \tau+\theta W_{\tau}\right. \\
& +\int_{0}^{\tau} \int_{-\infty}^{\infty}(\log (1+s)-s) \nu(d z) d s \\
& \left.+\int_{0}^{\tau} \int_{-\infty}^{\infty}(\log (1+s)-s)\left[N_{t}^{0}(d s, d z)+N_{t}^{i}(d s, d z)\right]\right\} \\
= & A_{t} \exp \left\{\left(\mu-\frac{1}{2} \theta^{2}\right) \tau+\varsigma_{0} W_{\tau}^{0}+\varsigma_{1} W_{\tau}^{i}\right. \\
& +\left[\frac{1}{1+\tau}-1-\frac{\tau^{2}}{2}\right]\left|\nu_{0}+\nu_{1}\right| \\
& \left.+\int_{0}^{\tau} \int_{-\infty}^{\infty}(\log (1+s)-s)\left[N_{t}^{0}(d s, d z)+N_{t}^{i}(d s, d z)\right]\right\}
\end{aligned}
$$

By putting $\tau=1$ and subtracting $\log B_{t}^{i}$ we get the Lemma.

In fact, the Lemma says that, as in the original model, the value of a borrower's assets depends on a common and an individual factors. However, the distributions of the factors are not the same as those of an increments of corresponding "driving" processes $U^{\bullet}$ as at the original model.

It remains to note that, generally, there are not closed formulas for distribution functions corresponding to variables $Y_{t}$ and $Z_{t}^{1}$ hence a $\mathrm{MC}$ simulation has to be used, possibly requiring special treatment, especially in the case of "jump" parts of the variables - for more on this topic, see [Cont and Tankov(2008)], Part II.

\section{Conclusion}

Summarized, we have formulated the dynamical version of the Merton-Vasicek model. Even if our results are not closed form, the model is tractable by simulation.

Acknoledgement: This work is supported by the grants no. 402/09/0965 and P402/10/1610 and P402/10/0956 of the Czech Science Foundation.

\section{References}

[Cont and Tankov(2008)] R. Cont and P. Tankov. Financial Modelling With Jump Processes. Chapman and Hall, Washington, 2008. ISBN 1-5848-8413-4.

[Hoffmann-Jørgenson(1994)] J. Hoffmann-Jørgenson. Probability with a View Towards to Statistics I. Chapman and Hall, New York, 1994. ISBN 0-4412-05231-8. 
[Kallenberg(2002)] O. Kallenberg. Foundations of Modern Probability. Springer, New York, second edition, 2002. ISBN 0-387-95313-2.

[O.A.Vasicek(2002)] O.A.Vasicek. The distribution of loan portfolio value,. RISK, 15:160-162, 2002.

[Oksendal and Sulem(2004)] B. Oksendal and A. Sulem. Look Inside This Book Applied Stochastic Control Of Jump Diffusions. Springer, NY, 2004. ISBN 3-540-14023-9.

[Pollard(2002)] D. Pollard. A User's Guide to Measure Theoretic Probability. Cambridge Univ. Press, Cambridge, 2002. ISBN 0-521-80242-3- 